Pacific Journal of Mathematics

ON THE OSCILLATION OF SOLUTIONS OF A CLASS OF 


\title{
ON THE OSCILLATION OF SOLUTIONS OF A CLASS OF LINEAR FOURTH ORDER DIFFERENTIAL EQUATIONS
}

\author{
SHAIR AHMAD
}

The purpose of this paper is to study the oscillation properties of the solutions of the differential equation

$$
y^{(4)}=p(t) y
$$

on $(-\infty, \infty)$, where $p(t)$ is positive and continuous for all $t$. Three different types of oscillation are considered, and necessary and sufficient conditions are given for the existence of solutions of each type.

Oscillatory behavior of solutions of (1) has been studied by a number of people (see [5]). Leighton and Nehari [3] have studied a slightly more general class of self-adjoint linear differential equations of fourth order and have given a number of results concerning oscillation of solutions of (1). Hastings and Lazer [2] have shown that under the additional assumptions $P \varepsilon C^{\prime}[a, \infty), p(t)>0$ and $p^{\prime}(t) \geqq 0$, (1) has two linearly independent oscillatory solutions which are bounded on $[a, \infty)$. If further, $\lim _{t \rightarrow \infty} p(t)=\infty$, then all oscillatory solutions of (1) tend to zero.

We shall distinguish between oscillation in the positive sense and that in the negative sense. A nontrivial solution of (1) is called positively (negatively) oscillatory if its set of zeros is not bounded above (below). It is called fully oscillatory if its set of zeros is neither bounded above nor below. It is called positively (negatively) (fully) nonoscillatory if it is not positively (negatively) (fully) oscillatory.

In order to gain some motivation, we consider the simple example

$$
y^{(4)}=y,
$$

whose solutions are of the form $y=c_{1} e^{x}+c_{2} e^{-x}+c_{3} \sin x+c_{4} \cos x$. We note that $y=e^{-x}+\sin x$ is a positively oscillatory solution of (2) which is not negatively oscillatory. Similarly, $y=\mathrm{e}^{x}+\sin x$ is a negatively oscillatory solution which is not positively oscillatory. Further, $y=\sin x$ and $y=\cos x$ are fully oscillatory.

We shall show that corresponding to the solutions $y=e^{x}$ and $y=e^{-x}$ of (2) there are solutions $w$ and $z$ of (1), respectively, such that $w(t), w^{\prime}(t), w^{\prime \prime}(t), w^{\prime \prime \prime}(t)>0$ for all $t \varepsilon(-\infty, \infty)$ and $z(t)>0$, $z^{\prime}(t)<0, \quad z^{\prime \prime}(t)>0$, and $z^{\prime \prime \prime}(t)<0$ for $t \varepsilon(-\infty, \infty)$. Under an additional assumption it is shown that any other solutions $w_{1}$ and $z_{1}$ 
with these properties must be constant multiples of $w$ and $z$, respectively. The main theorem concerning positive oscillation states that (1) has a positively oscillatory solution if and only if every positively nonoscillatory solution $y$ of (1) has the property that either there exists a number $t_{0}$ such that $\operatorname{sgn} y=\operatorname{sgn} y^{(j)}, j=1,2,3$, for all $t \geqq t_{o}$, or $\operatorname{sgn} y^{(j)} \neq \operatorname{sgn} y^{(j+1)}, j=0,1,2$, for all $t \varepsilon(-\infty, \infty)$. It then follows that if (1) has one positively oscillatory solution, it has three linearly independent positively oscillatory solutions. Two of these have the property that every solution which is a nontrivial linear combination of these two is also positively oscillatory. However, as suggested by the solutions of (2), given any three linearly independent solutions of (1), some linear combination of them is positively nonoscillatory. It is also shown that duals of the theorems concerning positively oscillatory solutions of (1) hold for the negatively oscillatory solutions. Finally, a necessary and sufficient condition is given for the existence of a fully oscillatory solution of (1) and it is shown that if (1) has one fully oscillatory solution, then it has two linearly independent fully oscillatory solutions such that the zeros of any two independent linear combinations of these solutions separate on $(-\infty, \infty)$. Further, any nontrivial linear combination of these two solutions is also fully oscillatory. As suggested by the solutions of (2), if $y_{1}$ and $y_{2}$ are any two independent linear combinations of these two solutions, then $y_{1}^{(i)}$ and $y_{2}{ }^{(i)}$ cannot have common zeros, $i=0,1,2,3$.

2. Positive oscillation. First we state two lemmas. See [2] and [3] for proofs.

LEMMA 1. If $y(t)$ is a nontrivial solution of (1) and $a$ is a number such that $y(a), y^{\prime}(a), y^{\prime \prime}(a), y^{\prime \prime \prime}(a) \geqq 0$, then $y(t), y^{\prime}(t), y^{\prime \prime}(t)$, $y^{\prime \prime \prime}(t)>0$ for all $t>a$.

LEMMA 2. If $y(t)$ is a nontrival solution of (1) and $a$ is number such that $y(a) \geqq 0, y^{\prime}(a) \leqq 0, y^{\prime \prime}(a) \geqq 0$ and $y^{\prime \prime \prime}(a) \leqq 0$, then $y(t)>0$, $y^{\prime}(t)<0, y^{\prime \prime}(t)>0$ and $y^{\prime \prime \prime}(t)<0$ for all $t<a$.

We note if $y$ is a solution of (1), then so is $-y$. Hence it follows from Lemma 1 that $y(a), y^{\prime}(a), y^{\prime \prime}(a), y^{\prime \prime \prime}(a) \leqq 0$ (but not all zero) implies $y(t), y^{\prime}(t), y^{\prime \prime}(t), y^{\prime \prime \prime}(t)<0$ for all $t>a$. Similarly, it follows from Lemma 2 that if $y$ is a nontrivial solution such that $y(a) \leqq 0$, $y^{\prime}(a) \geqq 0, y^{\prime \prime}(a) \leqq 0$ and $y^{\prime \prime \prime}(a) \geqq 0$, then $y(t)<0, y^{\prime}(t)>0, y^{\prime \prime}(t)<0$ and $y^{\prime \prime \prime}(t)>0$ for all $t<a$.

Throughout the remainder of this paper we let $z_{0}, z_{1}, z_{2}$, and $z_{3}$ denote solutions of (1) defined on $(-\infty, \infty)$ by the initial conditions 


$$
z_{i}{ }^{(j)}(0)=\delta_{i j}=\left\{\begin{array}{l}
0, i \neq j \\
1, i=j
\end{array}\right.
$$

for $i, j=0,1,2,3$.

THEOREM 1. There exists a solution $w$ of (1) such that $w(t), w^{\prime}(t)$, $w^{\prime \prime}(t), w^{\prime \prime \prime}(t)>0$ for all $t$.

Proof. For each natural number $n$, let $c_{o n}, c_{1 n}, c_{2 n}$ and $c_{3 n}$ be numbers satisfying

$$
\begin{aligned}
& c_{o n} z_{0}(-n)+c_{1 n} z_{1}(-n)+c_{2 n} z_{2}(-n)+c_{3 n} z_{3}(-n)=0, \\
& c_{o n} z_{0}^{\prime}(-n)+c_{1 n} z_{1}^{\prime}(-n)+c_{2 n} z_{2}^{\prime}(-n)+c_{3 n} z_{3}^{\prime}(-n)=0 \text {, } \\
& c_{0 n} z_{0}^{\prime \prime}(-n)+c_{1 n} z_{1}^{\prime \prime}(-n)+c_{2 n} z_{2}^{\prime \prime}(-n)+c_{3 n} z_{3}^{\prime \prime}(-n)=0 \text {, } \\
& c_{0 n} z_{0}^{\prime \prime \prime}(-n)+c_{1 n} z_{1}^{\prime \prime \prime}(-n)+c_{2 n} z_{2}^{\prime \prime \prime}(-n)+c_{3 n} z_{3}^{\prime \prime \prime}(-n)>0 \text {, } \\
& c_{0 n}^{2}+c_{1 n}^{2}+c_{2 n}^{2}+c_{3 n}^{2}=1 \text {. }
\end{aligned}
$$

Let $y_{n}=c_{0 n} z_{0}+c_{1 n} z_{1}+c_{2 n} z_{2}+c_{3 n} z_{3}$. The existence of numbers $c_{0 n}, c_{1 n}$, $c_{2 n}$, and $c_{3 n}$, satisfying the above conditions, is easy to verify. For one can choose such numbers satisfying the first three equations and the last equation. Since $z_{0}, z_{1}, z_{2}$ and $z_{3}$ are linearly independent, $y_{n}$ is a nontrivial solution of (1). It follows from the Uniqueness Theorem that $y_{n}^{\prime \prime \prime}(-n) \neq 0$. If $y_{n}^{\prime \prime \prime}(-n)<0$, then $-c_{0 n},-c_{1 n},-c_{2 n}$, and $-c_{3 n}$ satisfy the required conditions.

Since for each natural number $n, c_{0 n}^{2}+c_{1 n}^{2}+c_{2 n}^{2}+c_{3 n}^{2}=1$, there exists a sequence $n_{k}$ of natural numbers such that the sequences $\left\{c_{o n_{k}}\right\},\left\{c_{1 n_{k}}\right\},\left\{c_{2 n_{k}}\right\}$ and $\left\{c_{3 n_{k}}\right\}$ converge to numbers $c_{0}, c_{1}, c_{2}$ and $c_{3}$, respectively, satisfying

$$
c_{0}^{2}+c_{1}^{2}+c_{2}^{2}+c_{3}^{2}=1 .
$$

Let $w=c_{0} z_{0}+c_{1} z_{1}+c_{2} z_{2}+c_{3} z_{3}$. Now, suppose that $w^{(j)}\left(t_{0}\right)<0$ for some $j=0,1,2,3$, and for some number $t_{0}$. Then, since $\left\{y_{n_{k}}^{(j)}\left(t_{0}\right)\right\}$ converges to $w^{j}\left(t_{0}\right)$, there exists a natural number $N$ such that $y_{n_{k}}^{(j)}(t)<0$ for all $n_{k}>N$. But this leads to a contradiction since for $-n_{k}<t_{0}$, $y_{n_{k}}^{(j)}\left(t_{o}\right)>0$ by Lemma 1 , as $y_{n_{k}}^{(j)}\left(-n_{k}\right) \geqq 0$ for all $j=0,1,2,3$. This shows that $w^{(j)}(t) \geqq 0$ for all $j=0,1,2,3,4$, and all $t$. Further, since $w$ is a nontrivial solution, there is no number $\tau$ such that $w^{(j)}(\tau)=0$ for all $j=0,1,2,3$. Therefore, it follows that $w$ satisfies the requirements of the theorem.

THEOREM 2. There exists a solution $z(t)$ of $(1)$ such that $z(t)>0$, $z^{\prime}(t)<0, z^{\prime \prime}(t)>0$ and $z^{\prime \prime \prime}(t)<0$ for all $t$.

Proof. The proof is similar to that of Theorem 1. We modify 
each $y_{n}$ to satisfy the conditions $y_{n}(n)=y_{n}^{\prime}(n)=y_{n}^{\prime \prime}(n)=0$ and $y_{n}^{\prime \prime \prime}(n)<0$, and use Lemma 2.

Throughout this paper, $w$ and $z$ represent the solutions of (1) given in Theorems (1) and (2), respectively.

LEMMA 3. If $y$ is a positively nonoscillatory solution of (1) which has a zero in common with some positively oscillatory solution of (1), then there exists a number $t_{0}$ such that $y, y^{\prime}, y^{\prime \prime}$ and $y^{\prime \prime \prime}$ have the same. constant sign on $\left[t_{0}, \infty\right)$.

Proof. Since $y$ is a positively nonoscillatory solution of (1), there exists a number $t_{0}$ such that $y, y^{\prime}, y^{\prime \prime}$ and $y^{\prime \prime \prime}$ are each of constant sign on $\left[t_{0}, \infty\right)$. Let $u$ be a positively oscillatory solution and $\tau$ a number such that $u(\tau)=y(\tau)=0$. An application of Rolle's Theorem to the function $u / y$ shows that there exists a number $s>t_{0}$ such that

$$
u^{\prime}(s) y(s)-u(s) y^{\prime}(s)=0 \text {. }
$$

Hence there exist nonzero constants $c_{1}$ and $c_{2}$ satisfying

$$
\begin{aligned}
& c_{1} u(s)+c_{2} y(s)=0, \\
& c_{1} u^{\prime}(s)+c_{2} y^{\prime}(s)=0 .
\end{aligned}
$$

Let $Y(t)$ be the solution of (1) given by

$$
Y(t)=c_{1} u(t)+c_{2} y(t) .
$$

Since $u$ is positively oscillatory and $y$ is not, $Y \neq 0$. Thus we can not have $Y^{\prime \prime}(s)=Y^{\prime \prime \prime}(s)=0$. Further, $\operatorname{sgn} Y^{\prime \prime}(s)=\operatorname{sgn} Y^{\prime \prime \prime}(s)$. For, otherwise, $Y$ would be of constant sign to the left of $s$, by Lemma 2, contradicting $Y(\tau)=0$. Hence, $\operatorname{sgn} Y(t)=\operatorname{sgn} Y^{(j)}(t), j=1,2,3$, for all $t>s$, by Lemma 1 . In order to complete the proof of this lemma, it is sufficient to show that $\operatorname{sgn} y^{\prime \prime}(t)=\operatorname{sgn} y^{\prime \prime \prime}(t)$ on $\left[t_{0}, \infty\right)$. Suppose $\operatorname{sgn} y^{\prime \prime}(t) \neq \operatorname{sgn} y^{\prime \prime \prime}(t)$ on $\left[t_{0}, \infty\right)$. Then $y^{\prime \prime}$ is bounded on $\left[t_{0}, \infty\right)$. But this is impossible since $u$ is positively oscillatory and $\operatorname{sgn} Y^{\prime \prime \prime}(t)=\operatorname{sgn}$ $Y^{(4)}(t)$ for $t>s$ implies that $\lim _{t \rightarrow \infty}\left|Y^{\prime \prime}(t)\right|=\infty$. This completes the proof.

THEOREM 3. The following two conditions are equivalent:

(A) There exists a positively oscillatory solution of (1).

(B) If $y$ is any positively nonoscillatory solution of (1), then. either there exists a number $t_{0}$ such that

$$
\operatorname{sgn} y(t)=\operatorname{sgn} y^{(j)}(t), \quad j=1,2,3,
$$

for all $t \geqq t_{0}$, or 


$$
\operatorname{sgn} y^{(j)}(t) \neq \operatorname{sgn} y^{(j+1)}(t), \quad j=0,1,2,
$$

for all $t \varepsilon(-\infty, \infty)$.

Proof. Assume (A) holds, and let $y$ be a positively nonoscillatory solution. Then there exists a number $t_{0}$ such that $y, y^{\prime}, y^{\prime \prime}$ and $y^{\prime \prime \prime}$ are each of constant sign on $\left[t_{0}, \infty\right)$. We note that if $\operatorname{sgn} y^{(j)}(t) \neq \operatorname{sgn}$ $y^{(j+1)}(t), j=0,1,2$, for all $t \varepsilon\left(t_{0}, \infty\right]$, then $\operatorname{sgn} y^{(j)}(t) \neq \operatorname{sgn} y^{(j+1)}(t)$, $j=0,1,2$, for all $t \varepsilon(-\infty, \infty)$, by Lemma 2 . Let $u$ be a positively oscillatory solution and $s>t_{0}$ with $u(s)=0$. Let $c$ be a nonzero constant such that

$$
y(s)+c z(s)=0 .
$$

Let $Y=y+c z$. If $Y \equiv 0$, then $y$ satisfies condition (B). Suppose $Y \equiv 0$. Assume, without loss of generality that $y>0$ on $\left[t_{0}, \infty\right)$. It is easy to verify that if $y$ does not satisfy condition (B), then we must have $y, y^{\prime}, y^{\prime \prime}>0$ and $y^{\prime \prime \prime}<0$ on $\left[t_{0}, \infty\right)$. Thus, $\lim _{t \rightarrow \infty} y(t)=\infty$, and consequently, $Y$ is positively nonoscillatory since $z$ is bounded on $\left[t_{0}, \infty\right)$. Hence by Lemma 3 , there exists a number $t_{1}$ such that $\operatorname{sgn} Y=\operatorname{sgn} Y^{(j)}$, $j=1,2,3$, for $t>t_{1}$. It follows that $\lim _{t \rightarrow \infty}\left|Y^{\prime \prime}(t)\right|=\infty$ since $\operatorname{sgn} Y^{\prime \prime \prime}=$ $\operatorname{sgn} Y^{(4)}$ for $t>t_{1}$. But this is a contradiction since $\mathrm{y}^{\prime \prime}$ and $z^{\prime \prime}$ are both bounded on $\left[t_{0}, \infty\right)$, as $y^{\prime \prime} y^{\prime \prime \prime}<0$ and $z^{\prime \prime} z^{\prime \prime \prime}<0$. This completes the proof of the first half of Theorem 3.

In order to prove that (B) implies (A), we construct two linearly independent positively oscillatory solutions $u^{+}$and $v^{+}$similar to what was done in [2]. Some of the details have to be modified since we do not require $p(t)$ to satisfy the same hypothesis as in [2]. For the sake of completeness we go through the construction of $u^{+}$and $v^{+}$.

For each natural number $n$, let $b_{0 n}, b_{3 n}, c_{2 n}$ and $c_{3 n}$ be numbers satisfying

$$
\begin{gathered}
b_{0 n}^{2}+b_{3 n}^{2}=c_{2 n}^{2}+c_{3 n}^{2}=1, \\
b_{0 n} z_{0}(n)+b_{3 n} z_{3}(n)=0, \\
c_{2 n} z_{2}(n)+c_{3 n} z_{3}(n)=0 .
\end{gathered}
$$

Define $u_{n}(t)$ and $v_{n}(t)$ to be the solutions of (1) given by

$$
\begin{aligned}
& u_{n}(t)=b_{0 n} z_{0}(t)+b_{3 n} z_{3}(t), \\
& v_{n}(t)=c_{2 n} z_{2}(t)+c_{3 n} z_{3}(t) .
\end{aligned}
$$

By (3) there exists a sequence $\left\{n_{k}\right\}$ of natural numbers and numbers $b_{0}, b_{3}, c_{2}, c_{3}$ such that the sequences $\left\{b_{0 n_{k}}\right\},\left\{b_{3 n_{k}}\right\},\left\{c_{2 n_{k}}\right\}$, and $\left\{c_{3 n_{k}}\right\}$ converge to $b_{0}, b_{3}, c_{2}$ and $c_{3}$, respectively, where $b_{0}^{2}+b_{3}^{2}=c_{2}^{2}+c_{3}^{2}=1$. Let $u^{+}$and $v^{+}$be the solutions of (1) given by 


$$
\begin{aligned}
& u^{+}(t)=b_{0} z_{0}(t)+b_{3} z_{3}(t), \\
& v^{+}(t)=c_{2} z_{2}(t)+c_{3} z_{3}(t) .
\end{aligned}
$$

Suppose $u^{+}$is positively nonoscillatory. Since (B) holds and $\left(u^{+}\right)^{\prime}(0)=0$, there exists a number $t_{0}$ such that for all $t>t_{0}$,

$$
\operatorname{sgn} u^{+}(t)=\operatorname{sgn}\left(u^{+}\right)^{(j)}(t), j=1,2,3 .
$$

Let $\tau$ be any number greater than $t_{0}$. Since $\left\{u_{n_{k}}(\tau)\right\},\left\{u_{n_{k}}^{\prime}(\tau)\right\}, u_{n_{k}}^{\prime \prime}(\tau)$, and $u_{n_{k}}^{\prime \prime \prime}(\tau)$ converge to $u^{+}(\tau), u\left(^{+}\right)^{\prime}(\tau),\left(u^{+}\right)^{\prime \prime}(\tau)$, and $\left(u^{+}\right)^{\prime \prime \prime}(\tau)$, respectively, there exists a natural number $N$ such that

$$
\operatorname{sgn} u_{n_{k}}(\tau)=\operatorname{sgn} u_{n_{k}}^{(j)}(\tau), j=1,2,3,
$$

for all $n_{k}>N$. Hence, by Lemma 1 ,

$$
\operatorname{sgn} u_{n_{k}}(t)=\operatorname{sgn} u_{n_{k}}^{(j)}(t), j=1,2,3,
$$

for all $t>\tau$ and $n_{k}>N$. But this is a contradiction since $u_{n_{k}}\left(n_{k}\right)=0$ for all natural numbers $n_{k}$. Therefore, $u^{+}$is positively oscillatory. Simillarly, $v^{+}$is also positively oscillatory (note: $v^{+}(0)=0$ ). This completes the proof of Theorem 3 .

CoROLlaRY. If (1) has a positively oscillatory solution and $y$ is: any positively nonoscillatory solution, then either $\lim _{t \rightarrow \infty}|y(t)|=\infty$ or $\lim _{t \rightarrow-\infty}|y(t)|=\infty$.

REMARK. An argument, similar to the one given to show that $u^{+}$is positively oscillatory, can be given to show that any nontrivial linear combination of $u^{+}$and $v^{+}$is positively oscillatory.

We note that $u^{+}$and $v^{+}$are linearly independent since, otherwise, we would have $u^{+}=k z_{3}, k \neq 0$; contradicting the fact that $u^{+}$is positively oscillatory.

THEOREM 4. If (1) has one positively oscillatory solution, then it has three linearly independent positively oscillatory solutions.

Proof. The existence of one positively oscillatory solution implies. condition (B) of Theorem 3 and hence the existence of two linearly independent positively oscillatory solutions $u^{+}$and $v^{+}$, as constructed in the proof of Theorem 3. In order to find a third positively oscillatory solution, let $c$ be a nonzero constant satisfying

$$
u^{+}(0)+c z(0)=0 \text {. }
$$

Let $y^{+}(t)=u^{+}(t)+c z(t)$. Suppose $y^{+}$is positively nonoscillatory. Since 
condition (B) holds and $y^{+}(0)=0$, there exists a number $t_{0}$ such that for all $t>t_{0}$,

$$
\operatorname{sgn} y^{+}(t)=\operatorname{sgn}\left(y^{+}\right)^{(j)}(t), j=1,2,3 .
$$

Therefore, $\lim _{t \rightarrow \infty}\left|y^{+}(t)\right|=\infty$, which is a contradiction since $u^{+}$is positively oscillatory and $z$ is bounded on $\left[t_{0}, \infty\right)$. That $y^{+}, u^{+}$, and $v^{+}$ are linearly independent follows. For, otherwise, $z$ would be a linear combination of $u^{+}$and $v^{+}$and hence positively oscillatory.

REMARK. We note that in view of Theorems (3) and (4), condition (B) is equivalent to the existence of three linearly independent positively oscillatory solutions.

The following theorem gives a necessary condition for the essential uniqueness of the solution $z(t)$.

THEOREM 5. Suppose that (1) has no positively oscillatory solution. If $y$ is any solution satisfying

$$
\operatorname{sgn} y^{(j)}(t) \neq \operatorname{sgn} y^{(j+1)}(t), j=0,1,2 .
$$

for all $t$, then $y=c z$ for some constant $c$.

Proof. Suppose that $y$ and $z$ are linearly independent. Let $c$ be a constant such that $z(0)+c y(0)=0$. Let $Y=z+c y$. Since $Y$ is positively nonoscillatory, there exists a number $t_{0}$ such that none of $Y, Y^{\prime}, Y^{\prime \prime}$ and $Y^{\prime \prime \prime}$ change sign on $\left[t_{0}, \infty\right)$. Assume, without loss of generality that $Y$ and, hence, $Y^{(4)}$ are positive on $\left[t_{0}, \infty\right)$. We note that $Y$ is bounded on this interval since $y$ and $z$ are both bounded. Therefore, we must have $Y^{\prime \prime \prime}<0, Y^{\prime \prime}>0$ and $Y^{\prime}<0$ for all $t \geqq t_{0}$. But this contradicts the fact that $Y(0)=0$, thus proving the theorem.

THEOREM 6. Let $y_{1}, y_{2}$ and $y_{3}$ be any three linearly independent solutions of (1). If (1) has a positively oscillatory solution, then some linear combination of $y_{1}, y_{2}$ and $y_{3}$ is positively nonoscillatory.

Proof. It is easy to verify that $w, z, u^{+}$and $v^{+}$form a basis for the solutions of (1), where $u^{+}$and $v^{+}$are the positively oscillatory solutions of Theorem 3. For, $z$ is bounded on $[0, \infty)$ and $\lim _{t \rightarrow \infty}$ $w(t)=\infty$; while, $w$ is bounded on $(-\infty, 0]$ and $\lim _{t \rightarrow-\infty} z(t)=\infty$. Hence $y_{1}, y_{2}$ and $y_{3}$ can be written as

$$
\begin{aligned}
& y_{1}=a_{1} u^{+}+a_{2} v^{+}+a_{3} w+a_{4} z, \\
& y_{2}=b_{1} u^{+}+b_{2} v^{+}+b_{3} w+b_{4} z,
\end{aligned}
$$




$$
y_{3}=c_{1} u^{+}+c_{2} v^{+}+c_{3} w+c_{4} z \text {. }
$$

Let $d_{1}, d_{2}$ and $d_{3}$ be numbers (not all zero) satisfying

$$
\begin{aligned}
& d_{1} a_{1}+d_{2} b_{1}+d_{3} c_{1}=0, \\
& d_{1} a_{2}+d_{2} b_{2}+d_{3} c_{2}=0 .
\end{aligned}
$$

Then $y=d_{1} y_{1}+d_{2} y_{2}+d_{3} y_{3}$ is a linear combination of $w$ and $z$ and hence positively nonoscillatory.

3. Negative oscillation. We note that $y(t)$ is a negatively oscillatory solution of (1) if and only if $Y(t)$ is a positively oscillatory solution of

$$
Y^{(4)}=P Y,
$$

where $Y(t)=y(-t)$ and $P(t)=p(-t)$. Further,

$$
\operatorname{sgn} y(t)=\operatorname{sgn} y^{(j)}(t), j=1,2,3,
$$

for all $t \varepsilon\left(-\infty, t_{0}\right]$ (and hence for $t$, by Lemma 1 ) if and only if

$$
\operatorname{sgn} Y^{(j)}(t) \neq \operatorname{sgn} Y^{(j+1)}(t), j=0,1,2,
$$

for all $t \varepsilon\left[-t_{0}, \infty\right)$ (and hence for all $t$, by Lemma 2). Similarly,

$$
\operatorname{sgn} y^{(j)}(t) \neq \operatorname{sgn} y^{(j+1)}(t), j=0,1,2,
$$

for all $t \varepsilon\left(-\infty, t_{0}\right]$ if and only if

$$
\operatorname{sgn} Y(t)=\operatorname{sgn} Y^{(j)}(t), j=1,2,3,
$$

for all $t \varepsilon\left[-t_{0}, \infty\right)$.

Thus, one can reduce the study of negatively oscillatory solutions of (1) to that of positively oscillatory solutions. We now state the duals of the preceding theorems concerning positively oscillatory solutions for negatively oscillatory solutions. The proofs follow from the above observations and the results established in the previous section.

LEMMA 3 . If $y$ is a negatively nonoscillatory solution of (1) which has a zero in common with some negatively oscillatory solution of (1), then there exists a number $t_{0}$ such that

$$
\operatorname{sgn} y^{(j)}(t) \neq \operatorname{sgn} y^{(j+1)}(t), j=0,1,2,
$$

for all $t \varepsilon\left(-\infty, t_{0}\right]$.

THEOREM 3'. The following two conditions are equivalent: 
(A') There exists a negatively oscillatory solution of (1).

$\left(\mathrm{B}^{\prime}\right)$ If $y$ is a negatively nonoscillatory solution of (1), then either there exists a number $t_{0}$ such that

$$
\operatorname{sgn} \mathrm{y}^{(j)}(t) \neq \operatorname{sgn} y^{(j+1)}(t), j=0,1,2,
$$

for all $t \varepsilon\left(-\infty, t_{0}\right]$, or

$$
\operatorname{sgn} y=\operatorname{sgn} y^{(j)}, j=1,2,3,
$$

for all $t \varepsilon(-\infty, \infty)$.

THEOREM 4'. If (1) has one negatively oscillatory solution, then it has three linearly independent negatively oscillatory solutions.

THEOREM 5'. Suppose that (1) has no negatively oscillatory solution. If $y$ is any solution such that

$$
\operatorname{sgn} y(t)=\operatorname{sgn} y^{(j)}(t), j=0,1,2,
$$

for all $t$, then $y=c w$ for some constant $c$.

THEOREM $6^{\prime}$. Let $y_{1}, y_{2}$ and $y_{3}$ be any three linearly independent solutions of (1). If (1) has a negatively oscillatory solution, then some linear combination of $y_{1}, y_{2}$ and $y_{3}$ is negatively nonoscillatory.

\section{Full oscillation.}

THEOREM 7. The following two conditions are equivalent:

$\left(\mathrm{A}^{\prime \prime}\right)$ There exists a fully oscillatory solution of (1).

$\left(\mathrm{B}^{\prime \prime}\right)$ Conditions (B) and (B') both hold.

Prooof. The fact that $\left(\mathrm{A}^{\prime \prime}\right)$ implies $\left(\mathrm{B}^{\prime \prime}\right)$ follows from Theorems (3) and $\left(3^{\prime}\right)$. Suppose that $\left(\mathrm{B}^{\prime \prime}\right)$ holds. For each natural number $n$, let $c_{0 n}, c_{1 n}$ and $c_{2 n}$ be numbers satisfying

$$
\begin{aligned}
c_{0 n} z_{0}(n)+c_{1 n} z_{1}(n)+c_{2 n} z_{2}(n) & =0, \\
c_{0 n} z_{0}(-n)+c_{1 n} z_{1}(-n)+c_{2 n} z_{2}(-n) & =0, \\
c_{0 n}^{2}+c_{1 n}^{2}+c_{2 n}^{2} & =1 .
\end{aligned}
$$

Similarly, let $b_{1 n}, b_{2 n}$ and $b_{3 n}$ be numbers satisfying

$$
\begin{aligned}
b_{1 n} z_{1}(n)+b_{2 n} z_{2}(n)+b_{3 n} z_{3}(n) & =0, \\
b_{1 n} z_{1}(-n)+b_{2 n} z_{2}(-n)+b_{3 n} z_{3}(-n) & =0, \\
b_{1 n}^{2}+b_{2 n}^{2}+b_{3 n}^{2} & =1 .
\end{aligned}
$$

Define $U_{n}(t)$ and $V_{n}(t)$ to be the solutions of (1) given by 


$$
\begin{aligned}
& U_{n}(t)=c_{0 n} z_{0}(t)+c_{1 n} z_{1}(t)+c_{2 n} z_{2}(t), \\
& V_{n}(t)=b_{1 n} z_{1}(t)+b_{2 n} z_{2}(t)+b_{3 n} z_{3}(t) .
\end{aligned}
$$

There exist subsequences $\left\{c_{o n_{k}}\right\},\left\{c_{1 n_{k}}\right\}$ and $\left\{c_{2 n_{k}}\right\}$ of $\left\{c_{0 n}\right\},\left\{c_{1 n}\right\}$ and $\left\{c_{2 n}\right\}$, respectively, and numbers $c_{0}, c_{1}$ and $c_{2}$ such that

$$
\begin{gathered}
\lim _{n_{k} \rightarrow \infty}\left\{c_{0 n_{k}}\right\}=c_{0}, \lim _{n_{k} \rightarrow \infty}\left\{c_{1 n_{k}}\right\}=c_{1}, \quad \lim _{n_{k} \rightarrow \infty}\left\{c_{2 n_{k}}\right\}=c_{2}, \\
c_{0}^{2}+c_{1}^{2}+c_{2}^{2}=1 .
\end{gathered}
$$

Let $U(t)=c_{0} z_{0}(t)+c_{1} z_{1}(t)+c_{2} z_{2}(t)$. One can verify, using an argument similar to the ones used in proofs of Theorems $(3)$ and $\left(3^{\prime}\right)$, that $U$ is fully oscillatory.

The solutions $V_{n}(t)$ give rise to a solution $V(t)$ of the form

$$
V(t)=b_{1} z_{1}(t)+b_{2} z_{2}(t)+b_{3} z_{3}(t),
$$

which is fully oscillatory. Similarly, any linear combination of $U$ and $V$ is also fully oscillatory.

REMARK. The solutions $U$ and $V$ of Theorem 7 are linearly independent. To see this it is sufficient to show that $c_{0} \neq 0$. Suppose $c_{0}=0$, so that $U=c_{1} z_{1}+c_{2} z_{2}$. Since $U(0)=U^{\prime \prime \prime}(0)=0$, either $\operatorname{sgn} U^{(j)}=\operatorname{sgn} U^{(j+1)}$, $j=1,2,3$, for all $t>0$, or $\operatorname{sgn} U^{(j)} \neq \operatorname{sgn} U^{(j+1)}, j=0,1,2$, for all $t<0$. Hence $U$ is not fully oscillatory, a contradiction.

THEOREM 8. Let $U$ and $V$ be the solutions of Theorem 7. If $Y_{1}$ and $Y_{2}$ are two independent linear combinations of $U$ and $V$, then $Y_{1}^{(j)}$ and $Y_{2}^{(j)}$ cannot have any common zeros, $j=0,1,2,3$.

Proof. Suppose that $Y_{1}^{(j)}(s)=Y_{2}^{(j)}(s)=0$ for some $j=0,1,2$, and some number $s$. Then there exist nonzero constants $c_{1}$ and $c_{2}$ satisfying

$$
\begin{aligned}
c_{1} Y_{1}^{(j)}(s)+c_{2} Y_{2}^{(j)}(s) & =0, \\
c_{1} Y_{1}^{(j+1)}(s)+c_{2} Y_{2}^{(j+1)}(s) & =0 .
\end{aligned}
$$

Let $Y=c_{1} Y_{1}+c_{2} Y_{2}$. Then by Lemmas (1) and (2), $Y$ is either positively or negatively nonoscillatory; contradicting the fact that every linear combination of $U$ and $V$ is fully oscillatory. If $j=3$, the proof is similar; we replace (7) by

$$
c_{1} Y_{1}^{(j-1)}(s)+c_{2} Y_{2}^{(j-1)}(s)=0 .
$$

THEOREM 9. If (1) has one fully oscillatory solution, then it has two independent fully oscillatory solutions $U$ and $V$ such that every nontrivial linear combination of $U$ and $V$ is fully oscillatory, and 
the zeros of any two independent linear combinations of $U$ and $V$ separate on $(-\infty, \infty)$. Further, $U, V, z$ and $w$ form $a$ basis for the solutions of $(1)$.

Proof. We simply prove that the zeros of any two independent linear combinations $Y_{1}$ and $Y_{2}$ of $U$ and $V$ separate on $(-\infty, \infty)$, since the remaining assertions are now obvious. Assuming that $U$ and $V$ are the solutions of Theorem 7 , let $t_{1}$ and $t_{2}\left(t_{1}<t_{2}\right)$ be any two consecutive zeros of $Y_{1}$. Suppose that $Y_{2}$ has no zero between $t_{1}$ and $t_{2}$. Then by Theorem $8, Y_{2}$ does not vanish in the interval $\left[t_{1}, t_{2}\right]$. Thus, $\left(Y_{1} / Y_{2}\right)^{\prime}$, and hence $Y_{2} Y_{1}^{\prime}-Y_{1} Y_{2}^{\prime}$, vanishes at some point $s$ between $t_{1}$ and $t_{2}$. Therefore, there exist nonzero constants $c_{1}$ and $c_{2}$ satisfying

$$
\begin{aligned}
c_{1} Y_{1}(s)+c_{2} Y_{2}(s) & =0, \\
c_{1} Y_{1}^{\prime}(s)+c_{2} Y_{2}^{\prime}(s) & =0 .
\end{aligned}
$$

But this leads to a contradiction, since the solution $y=c_{1} Y_{1}+c_{2} Y_{2}$ cannot be fully oscillatory by Lemmas (1) and (2).

REMARK. Since $U, V, w$ and $z$ form a basis for the solutions of (1), it follows that any fully oscillatory solution of (1) which is bounded on $(-\infty, \infty)$ must be a linear combination of $U$ and $V$. One might conjecture that every fully oscillatory solution is a linear combination of $U$ and $V$. We note that this is not true for positively or negatively oscillatory solutions. In fact, $y=\sin x+e^{-x}$ is a positively oscillatory solution of (2) which is not a linear combination of $\sin x$ and $\cos x$.

\section{REFERENCES}

1. W. B. Fite, Concerning the zeros of the solutions of certain differential equations, Trans. Amer. Math. Soc. 19 (1917), 341-352.

2. S. P. Hastings and A. C. Lazer, On the asymptotic behavior of solutions of the differential equation $y^{(4)}=p(t) y$, Czech. Math. J. 93 (1968), 224-229.

3. Walter Leighton and Zeev Nehari, On the oscillation of solutions of self-adjoint linear differential equations of the fourth order, Trans. Amer. Math. Soc. 89 (1958), 325-377.

4. M. Svec, Sur les dispersions de l'equation $y^{i v}+Q(x) y=0$, Czech. Math. J. 5 (1955), 29-60.

5. C. A. Swanson, Comparison and oscillation theory of linear differential equations, Academic Press, New York, 1968.

Receiveed January 16, 1970.

OKLAhoma State University 



\title{
PACIFIC JOURNAL OF MATHEMATICS
}

\author{
EDITORS
}

\author{
H. SAMELSON \\ Stanford University \\ Stanford, California 94305 \\ RichaRd PIERCE \\ University of Washington \\ Seattle, Washington 98105
}

J. DugundJI

Department of Mathematics

University of Southern California

Los Angeles, California 9.0007

RICHARD ARENS

University of California

Los Angeles, California 9.0024

\section{ASSOCIATE EDITORS}

E. F. BeCKenBACH
B. H. NeumanN

F. WoLE

K. YoSHIDA

\section{SUPPORTING INSTITUTIONS}

\author{
UNIVERSITY OF BRITISH COLUMBIA \\ CALIFORNIA INSTITUTE OF TECHNOLOGY \\ UNIVERSITY OF CALIFORNIA \\ MONTANA STATE UNIVERSITY \\ UNIVERSITY OF NEVADA \\ NEW MEXICO STATE UNIVERSITY \\ OREGON STATE UNIVERSITY \\ UNIVERSITY OF OREGON \\ OSAKA UNIVERSITY \\ UNIVERSITY OF SOUTHERN CALIFORNIA
}

\author{
STANFORD UNIVERSITY \\ UNIVERSITY OF TOKYO \\ UNIVERSITY OF UTAH \\ WASHINGTON STATE UNIVERSITY \\ UNIVERSITY OF WASHINGTON \\ AMERICAN MATHEMATICAL SOCIETY \\ CHEVRON RESEARCH CORPORATION \\ TRW SYSTEMS \\ NAVAL WEAPONS CENTER
}

The Supporting Institutions listed above contribute to the cost of publication of this Journal, but they are not owners or publishers and have no responsibility for its content or policies.

Mathematical papers intended for publication in the Pacific Journal of Mathematics should be in typed form or offset-reproduced, (not dittoed), double spaced with large margins. Underline Greek letters in red, German in green, and script in blue. The first paragraph or two must be capable of being used separately as a synopsis of the entire paper. The editorial "we" must not be used in the synopsis, and items of the bibliography should not be cited there unless absolutely necessary, in which case they must be identified by author and Journal, rather than by item number. Manuscripts, in duplicate if possible, may be sent to any one of the four editors. Please classify according to the scheme of Math. Rev. Index to Vol. 39. All other communications to the editors should be addressed to the managing editor, Richard Arens, University of California, Los Angeles, California, 90024.

50 reprints are provided free for each article; additional copies may be obtained at cost in multiples of 50 .

The Pacific Journal of Mathematics is published monthly. Effective with Volume 16 the price per volume (3 numbers) is $\$ 8.00$; single issues, $\$ 3.00$. Special price for current issues to individual faculty members of supporting institutions and to individual members of the American Mathematical Society: $\$ 4.00$ per volume; single issues $\$ 1.50$. Back numbers are available.

Subscriptions, orders for back numbers, and changes of address should be sent to Pacific Journal of Mathematics, 103 Highland Boulevard, Berkeley, California, 94708.

PUBLISHED BY PACIFIC JOURNAL OF MATHEMATICS, A NON-PROFIT CORPORATION

Printed at Kokusai Bunken Insatsusha (International Academic Printing Co., Ltd.), 7-17, Fujimi 2-chome, Chiyoda-ku, Tokyo, Japan. 


\section{Pacific Journal of Mathematics}

\section{Vol. 34, No. $2 \quad$ June, 1970}

Shair Ahmad, On the oscillation of solutions of a class of linear fourth order

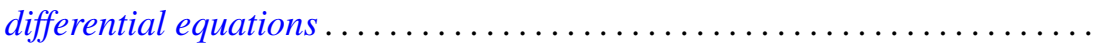

Leonard Asimow and Alan John Ellis, Facial decomposition of linearly

compact simplexes and separation of functions on cones ..............

Kirby Alan Baker and Albert Robert Stralka, Compact, distributive lattices of

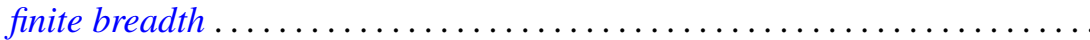

James W. Cannon, Sets which can be missed by side approximations to

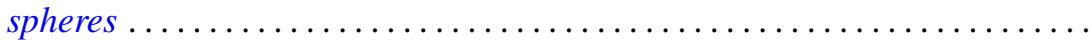

Prem Chandra, Absolute summability by Riesz means .................. 335

Francis T. Christoph, Free topological semigroups and embedding topological semigroups in topological groups....

Henry Bruce Cohen and Francis E. Sullivan, Projecting onto cycles in smooth, reflexive Banach spaces.................................

John Dauns, Power series semigroup rings .......................

Robert E. Dressler, A density which counts multiplicity ................

Kent Ralph Fuller, Primary rings and double centralizers ................

Gary Allen Gislason, On the existence question for a family of products.......

Alan Stuart Gleit, On the structure topology of simplex spaces .............

William R. Gordon and Marvin David Marcus, An analysis of equality in

certain matrix inequalities. $I \ldots \ldots \ldots \ldots \ldots \ldots \ldots \ldots$

Gerald William Johnson and David Lee Skoug, Operator-valued Feynman

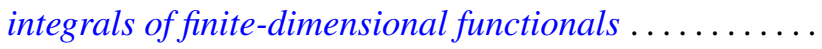

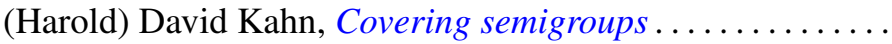

Keith Milo Kendig, Fibrations of analytic varieties

Norman Yeomans Luther, Weak denseness of nonatomic measures on perfect,

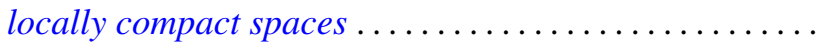

Guillermo Owen, The four-person constant-sum games; Discriminatory solutions on the main diagonal ...

Stephen Parrott, Unitary dilations for commuting contractions

Roy Martin Rakestraw, Extremal elements of the convex cone $A_{n}$ of

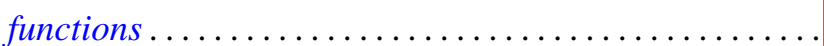

Peter Lewis Renz, Intersection representations of graphs by

William Henry Ruckle, Representation and series summability of complete

biorthogonal sequences.

F. Dennis Sentilles, The strict topology on bounded sets ...

Saharon Shelah, A note on Hanf numbers ...

Harold Simmons, The solution of a decision problem for several classes of rings. . .

Kenneth S. Williams, Finite transformation formulae involving the Legendre 